\title{
Numerical Study of the Flow of Two Radiative Nanofluids with Marangoni Convection Embedded in Porous Medium
}

\author{
Ravi Gupta $\mathbb{D}^{1},{ }^{1}$ Manish Gaur $\mathbb{D}^{2},{ }^{2}$ Qasem Al-Mdallal $\mathbb{D}^{3}$, Sunil Dutt Purohit $\mathbb{D},{ }^{4}$ \\ and Daya Lal Suthar $\mathbb{D}^{5}$ \\ ${ }^{1}$ School of Science and Technology, Vardhman Mahaveer Open University, Kota, India \\ ${ }^{2}$ Department of Mathematics, Government PG College, Kota, India \\ ${ }^{3}$ Department of Mathematical Sciences, UAE University, P.O. Box 15551, Al Ain, UAE \\ ${ }^{4}$ Department of HEAS (Mathematics), Rajasthan Technical University, Kota 324010, India \\ ${ }^{5}$ Department of Mathematics, Wollo University, P.O. Box 1145, Dessie, Ethiopia
}

Correspondence should be addressed to Daya Lal Suthar; dlsuthar@gmail.com

Received 19 October 2021; Revised 28 December 2021; Accepted 30 December 2021; Published 17 January 2022

Academic Editor: Taza Gul

Copyright (C) 2022 Ravi Gupta et al. This is an open access article distributed under the Creative Commons Attribution License, which permits unrestricted use, distribution, and reproduction in any medium, provided the original work is properly cited.

\begin{abstract}
The aim of this study is to investigate the flow of two distinct nanofluids over a stretching surface in a porous medium with Marangoni convection. This investigation is studied under the effect of thermal radiation. Here, we have considered $\mathrm{Fe}_{3} \mathrm{O}_{4}$ and $\mathrm{ZrO}_{2}$ nanosized particles suspended in engine oil (EO) base fluid. For the numerical simulation of the flow, the fourth-order Runge-Kutta method and suitable similarity solutions were used. Numerical solutions with graphical representation are presented. $\mathrm{Fe}_{3} \mathrm{O}_{4} / \mathrm{EO}$ nanofluid is more significant in the cooling process in comparison to $\mathrm{ZrO}_{2} / \mathrm{EO}$ nanofluid. With increased radiation and temperature ratio parameters, a decrement in the temperature field has been noticed for both nanofluids. For increased values of volume friction parameter, a decrement is noticed for velocity profile and increment is noted for temperature profiles for both nanofluids. Also, a reduced velocity profile can be obtained with increased porosity parameter.
\end{abstract}

\section{Introduction}

In the past two decades, nanofluids got the vast attention of researchers due to their proven efficiency in thermal conductivity. A base fluid with suspended nanometer-sized particles with different shapes and sizes is called nanofluids. These nanoparticles can be metallic or nonmetallic or oxidic, for example, $\mathrm{Al}, \mathrm{Cu}, \mathrm{Ag}, \mathrm{SiO}_{2}, \mathrm{Al}_{2} \mathrm{O}_{3}, \mathrm{Fe}_{3} \mathrm{O}_{4}$, and $\mathrm{ZrO}_{2}$, and base fluids can be $\mathrm{H}_{2} \mathrm{O}, \mathrm{C}_{2} \mathrm{H}_{6} \mathrm{O}_{2}$, and engine oil. Initially, Choi [1] investigated this phenomenon and named these fluids as "nanofluids." Afterward, Xuan and Roetzel [2] studied the correlation between heat transfer and nanofluids and presented a numerical model. The heat transfer capacity of nanofluids is much higher than that of conventional fluid because the thermal conductivity of a fluid can be optimized by adding nanosized particles; a number of studies have been presented by researchers proving this concept for the nanofluids using different sizes, shapes, and concentrations of nanoparticles with different base liquids, on different geometries [3-13]. Some key applications of nanofluids are in the field of nuclear plants, micropolymer films, heat exchangers, electronic devices, space technology, and production of heat pipes.

The convection characterized by the surface tension differences at the interface is defined as Marangoni convection. This interface dissipative flow can be relevant where different surface tension exists at interfaces, and by changing the temperature or concentration, this difference can be created. Marangoni convective boundary layer flows of nanoliquids have their applications in various fields like thin film, melting of crystals, welding, semiconductors, vapor bubbles, material sciences, soap films, silicon wafers, and microgravity conditions. Napolitano [14] was the first who gave this phenomenon and named it. Christopher and Wang [15] studied the Prandtl number effects for Marangoni convective flow through a flat surface. Furthermore, Aly and Ebaid [16] 
investigated radiative Marangoni convective flow of three different hybrid nanofluids with porosity and MHD effects. The thermal performance of glycerol/water mixture in the microchannel with Marangoni convection is studied by Yang et al. [17]. Afterward, this phenomenon is studied with different parameters, nanofluids, and geometries by some researchers [18-20].

Although nanofluids have been studied by many researchers with different nanoparticles suspended in different base fluids, in this study, engine oil base fluid is considered which is rare in the literature. Marangoni convection is an important aspect of determining fluid motion and material processing, particularly in low-gravity hydrodynamics, for analyzing heat transfer interactions, and nanoparticles can improve the flow's thermal performance. As per the best knowledge of the authors, the present study of Marangoni convective radiative flow of nanosized particles suspended in engine oil (EO) base fluid is novel. A comparative analysis for both nanofluids is also presented by the graphs and discussed and obtained a strong agreement with the existing literature.

\section{Mathematical Model}

Marangoni convective boundary layer flow of two different viscous, compressible nanofluids with thermal radiation, along the stretched surface, is studied in 2D. Engine oil is considered the base fluid with suspension of $\mathrm{Fe}_{3} \mathrm{O}_{4}$ and $\mathrm{ZrO}_{2}$ nanoparticles. Base fluid and nanosized particles of laminar flow are assumed to be thermally stable. The fluid flow is considered at $y \geq 0$; here, the $x$-axis is chosen parallel to the surface, and the $y$-axis is considered perpendicular to the stretching surface. $T_{w}$ is the temperature at the surface, and $T_{\infty}$ is the ambient temperature. Likewise, as Marangoni convection is considered, a linear relation of surface tension with temperature is given by [19]

$$
\gamma=\gamma_{0}\left[1-\bar{\gamma}\left(T-T_{\infty}\right)\right]
$$

Here, $\gamma_{0}$ is the surface tension and $\bar{\gamma}$ is the rate of change of surface tension along $T$ (temperature). Taking these assumptions, the equation of the governing convective flows of the nanofluid is described as follows [19]:

$$
\begin{gathered}
\frac{\partial u}{\partial x}+\frac{\partial v}{\partial y}=0 \\
u \frac{\partial u}{\partial x}+v \frac{\partial v}{\partial y}=\frac{\mu_{\mathrm{nf}}}{\rho_{\mathrm{nf}}} \frac{\partial^{2} u}{\partial y^{2}}-\frac{\mu_{\mathrm{nf}}}{\rho_{\mathrm{nf}} k} \\
u \frac{\partial T}{\partial x}+v \frac{\partial T}{\partial y}=\frac{k_{\mathrm{nf}}}{\left(\rho C_{p}\right)_{\mathrm{nf}}} \frac{\partial^{2} T}{\partial y^{2}}-\frac{1}{\left(\rho C_{p}\right)_{\mathrm{nf}}}\left(\frac{\partial q_{\mathrm{r}}}{\partial y}\right),
\end{gathered}
$$

with the boundary condition:

$$
\begin{gathered}
v=0, T=T_{\infty}+a x^{2}, \mu_{\mathrm{nf}}\left(\frac{\partial u}{\partial y}\right)=\frac{\partial \sigma}{\partial T} \frac{\partial T}{\partial x} \text { at } y=0, \\
u \longrightarrow 0, T \longrightarrow T_{\infty} \text { as } y \longrightarrow \infty
\end{gathered}
$$

Here, velocity notations in $x$ and $y$ directions are chosen as $u$ and $v$ accordingly. Radiative heat flux is represented by $q_{\mathrm{r}}$. The Rosseland approximation takes into account radiative heat flux which is reported by [20]

$$
q_{\mathrm{r}}=-\frac{4 \sigma^{*}}{3 k^{*}} \frac{\partial T^{4}}{\partial y}=-\frac{16 \sigma^{*}}{3 k^{*}} T^{3} \frac{\partial T}{\partial y}
$$

In the above equation, the mean absorption coefficient is denoted by $k^{*}$ and $\sigma^{*}$ denotes the Stefan-Boltzmann constant. After substituting this $q_{\mathrm{r}}$ into equation (4), the reduced equation is given as follows:

$$
u \frac{\partial T}{\partial x}+v \frac{\partial T}{\partial y}=\frac{k_{\mathrm{nf}}}{\left(\rho C_{p}\right)_{\mathrm{nf}}} \frac{\partial^{2} T}{\partial y^{2}}+\frac{16 \sigma^{*}}{3\left(\rho C_{p}\right)_{\mathrm{nf}} k^{*}} \frac{\partial}{\partial y}\left(T^{3} \frac{\partial T}{\partial y}\right)
$$

Here, $\mu_{\mathrm{nf}}$ is the viscosity, $\rho_{\mathrm{nf}}$ is the density, $\kappa_{\mathrm{nf}}$ is the thermal conductivity, and $\left(\rho C_{p}\right)_{\text {nf }}$ is the heat capacity of nanofluids. Subscripts $\mathrm{f}$ and $\mathrm{nf}$ are used to denote base fluids and nanofluids accordingly. Also, the volume fraction is $\phi$; also, $m=3$ is chosen for spherical-type nanoparticles. Physical constraints and thermophysical value of nanoliquids and base fluid are reported in Tables 1 and 2, respectively.

\section{Similarity Solutions}

The following similarity transformation is used to solve our model [19]:

$$
\eta=\zeta_{1} y, \varphi=\zeta_{2} x f(\eta), \theta(\eta)=\frac{T-T_{\infty}}{T_{w}-T_{\infty}}
$$

where $\zeta_{1}=\left(\gamma_{0} \bar{\gamma} a \rho_{\mathrm{f}} / \mu_{\mathrm{f}}^{2}\right)^{1 / 3}, \zeta_{2}=\left(\gamma_{0} \bar{\gamma} a \mu_{\mathrm{f}} / \rho_{\mathrm{f}}^{2}\right)^{1 / 3}, u=\partial \varphi / \partial y$, and $v=-\partial \varphi / \partial x$.

Following the foregoing transformations, equations (3) and (7) are turned into nonlinear ordinary differential equations as follows:

$$
\begin{aligned}
f^{\prime \prime \prime}+\left(1-\phi+\phi \frac{\rho_{s}}{\rho_{\mathrm{f}}}\right)(1-\phi)^{2.5}\left(f f^{\prime \prime}-f^{\prime 2}\right)-K f^{\prime}=0 \\
\theta^{\prime \prime}\left[1+R_{d} A\left(\theta\left(\theta_{w}-1\right)+1\right)^{3}\right] \\
+R_{d} A\left[3 \theta^{\prime 2}\left(\theta_{w}-1\right)\left\{\theta\left(\theta_{w}-1\right)+1\right\}^{2}\right] \\
+B\left(f \theta^{\prime}-2 \theta f^{\prime}\right)=0
\end{aligned}
$$

Here, $A=\kappa_{\mathrm{f}} / \kappa_{\mathrm{nf}}=\left\{\left(\kappa_{s}+(m-1) \kappa_{\mathrm{f}}+\left(\kappa_{\mathrm{f}}-\kappa_{s}\right) \phi\right) /\left(\kappa_{s}+(m\right.\right.$ $\left.\left.-1) \kappa_{\mathrm{f}}-(m-1)\left(\kappa_{\mathrm{f}}-\kappa_{s}\right) \phi\right)\right\}, \quad B=\operatorname{Pr}_{\mathrm{f}}\left(1-\phi+\phi\left(\rho_{s} / \rho_{\mathrm{f}}\right)\right)$ $(1-\phi)^{2.5}$, and $C=(1-\phi)^{2.5}$. 
TABLE 1: Table of physical constraints of nanofluids used.

$$
\begin{aligned}
& \mu_{\mathrm{nf}}=\mu_{\mathrm{f}} /(1-\phi)^{2.5} \\
& \rho_{\mathrm{nf}}=(1-\phi) \rho_{\mathrm{f}}+\phi \rho_{s} \\
& \kappa_{\mathrm{nf}}=\left\{\kappa_{s}+(m-1) \kappa_{\mathrm{f}}-(m-1)\left(\kappa_{\mathrm{f}}-\kappa_{s}\right) \phi / \kappa_{s}+(m-1) \kappa_{\mathrm{f}}+\left(\kappa_{\mathrm{f}}-\kappa_{s}\right) \phi\right\} \kappa_{\mathrm{f}} \\
& \quad\left(\rho C_{p}\right)_{\mathrm{nf}}=\left(\rho C_{p}\right)_{s} \phi+\left(\rho C_{p}\right)_{\mathrm{f}}(1-\phi)
\end{aligned}
$$

Effective dynamic viscosity

Effective density

Thermal conductivity

Heat capacitance
The above equations are with the modified boundary conditions:

$$
\theta(0)=1, f(0)=0, f^{\prime \prime}(0)=-2 C, \theta(\infty)=0, f^{\prime}(\infty)=0,
$$

where $R_{d}=16 \sigma^{*} T_{\infty}^{3} / 3 k^{*} k_{f}$ is the radiation parameter, $\operatorname{Pr}=\left(\rho C_{p}\right)_{\mathrm{f}} v_{\mathrm{f}} / \kappa_{\mathrm{f}}$ is the Prandtl number, $K=1 / \psi_{1}^{2} k$ is the permeability parameter, and $\theta_{w}=T_{w} / T_{\infty}$ is the temperature ratio parameter.

\section{Numerical Solution}

With the help of the above-described similarity transformation, the governing equation of the flows has been converted into a set of the ordinary differential equations. Moreover, in order to tackle these equations using the Runge-Kutta method with a shooting technique, equations (9) and (10) together with the boundary conditions (11) turned into an initial value problem, as shown below.

$f=h_{1}, f^{\prime}=h_{2}, f^{\prime \prime}=h_{3}, \theta=h_{4}$, and $\theta^{\prime}=h_{5}$, where $h_{3}{ }^{\prime}=$ $(1-\phi)^{2.5}\left(1-\phi+\phi\left(\rho_{s} / \rho_{\mathrm{f}}\right)\right)\left(h_{2}^{2}-h_{1} h_{3}\right)+K h_{2}$ and $h_{5}^{\prime}=\left(-R_{d}\right.$ $\left.A\left[3 h_{5}^{2}\left(\theta_{w}-1\right)\left\{h_{4}\left(\theta_{w}-1\right)+1\right\}^{2}\right]-B\left(h_{1} h_{5}-2 h_{4} h_{2}\right)\right) /\left(1+R_{d}\right.$ $\left.A\left\{h_{4}\left(\theta_{w}-1\right)+1\right\}^{3}\right)$.

The above equations are with the following transformed boundary conditions, $h_{1}(0)=0, h_{3}(0)=-2 C$, and $h_{4}(0)=1$. The best approximated numerical results are obtained by some initial guesses with the shooting method. To get exact results up to $10^{-7}$ accuracy, an iterated approach is used in this method. The Prandtl number for EO is 6450, and the step size $\Delta \eta=0.01$ is considered.

\section{Results and Discussion}

The comparative investigation of Marangoni convection of $\mathrm{Fe}_{3} \mathrm{O}_{4}$ and $\mathrm{ZrO}_{2}$ nanofluid flow is studied. This investigation is studied under the effect of thermal radiation over the stretching surface. Numerical simulation is performed with the above-described method, and results are presented graphically to illustrate the impacts of several nondimensional physical parameters for $f^{\prime}(\eta)$ (temperature profile) and $\theta(\eta)$ (velocity profile).

The impact of $K$ on $f^{\prime}(\eta)$ for $\mathrm{Fe}_{3} \mathrm{O}_{4} / \mathrm{EO}$ and $\mathrm{ZrO}_{2} / \mathrm{EO}$ nanofluids, respectively, is depicted in Figure 1. It is observed that with increased $K$, a decrement in $f^{\prime}(\eta)$ is seen for both nanofluids. With the fact $K \propto 1 / k$, hence if we raise the coefficient of porosity, the permeability of the porous media decreases, resulting in a drop in fluid velocity.
TABLE 2: Thermophysical properties $[19,20]$.

\begin{tabular}{lcccc}
\hline & $\rho\left(\mathrm{kg} \mathrm{m}^{-3}\right)$ & $C_{p}\left(\mathrm{~J} \mathrm{~kg}^{-1} \mathrm{~K}^{-1}\right)$ & $k\left(\mathrm{~W} \mathrm{~m}^{-1} \mathrm{~K}^{-1}\right)$ & $\mathrm{Pr}$ \\
\hline $\mathrm{EO}$ & 884 & 1910 & 0.144 & 6450 \\
$\mathrm{Fe}_{3} \mathrm{O}_{4}$ & 5810 & 670 & 6 & - \\
$\mathrm{ZrO}_{2}$ & 5680 & 502 & 1.7 & - \\
\hline
\end{tabular}

The effect of volume friction $\phi$ on $f^{\prime}(\eta)$ is depicted in Figure 2. It is noticed that with increased $\phi$, we get a decreased velocity profile. With rising $\phi$, there is a significant influence of $\phi$ on the velocity profiles, with the speed of fluid being reduced at the vicinity of the surface and the reverse effect being visible further from the surface. It is also worth noting that the $\mathrm{ZrO}_{2} / \mathrm{EO}$ nanofluid has a larger velocity than the $\mathrm{Fe}_{3} \mathrm{O}_{4} / \mathrm{EO}$ nanofluid. This is due to the fact that density of $\mathrm{ZrO}_{2}$ is lower than that of $\mathrm{Fe}_{3} \mathrm{O}_{4}$, implying that $\mathrm{ZrO}_{2}$ is lighter in motion than $\mathrm{Fe}_{3} \mathrm{O}_{4}$.

The impact of $\phi$ on $\theta(\eta)$ is depicted in Figure 3. It is observed that with increased $\phi$, an increased temperature profile for both nanofluids is seen. With increased nanoparticles of both nanofluids, the temperature of fluid is increased; as a result, the temperature field may rise.

The impact of $R_{d}$ on $\theta(\eta)$ is depicted in Figure 4. It is concluded that with increased $R_{d}$ along with $\theta(\eta)$, an increased temperature profile is observed. Radiations create additional heat within the fluids, which results in higher temperature fields. Furthermore, $R_{d}$ has no substantial effect on the velocity fields. The radiation parameter, which is the inverse of the Stephan number, is used to determine the relative significance of thermal radiation versus conductive heat transmission. As a result of the thermal radiation's dominance over conduction, it may be employed to regulate the thermal boundary layers rather well.

The impact of the temperature ratio parameter $\theta_{w}$ on $\theta(\eta)$ is depicted in Figure 5. It is concluded that with increased $\theta_{w}$ along with $\theta(\eta)$, an increased temperature profile is observed. As a recognizable outcome, it is likewise seen that the temperature profile of the $\mathrm{Fe}_{3} \mathrm{O}_{4} / \mathrm{EO}$ nanoliquid remains consistently greater than that of the $\mathrm{ZrO}_{2} / \mathrm{EO}$ nanoliquid.

\section{Code of Verification}

For the validation of this research, comparison with Das et al. [21] and Jamshed et al. [4] was made for local Nusselt numbers using several values of $\operatorname{Pr}$ as shown in Table 3. To do this, the remaining parameters in our simulation approach are set to zero $\left(R_{d}=K=\phi=\theta_{w}=0\right)$. The calculated findings are found to be in good match with the previous results. 


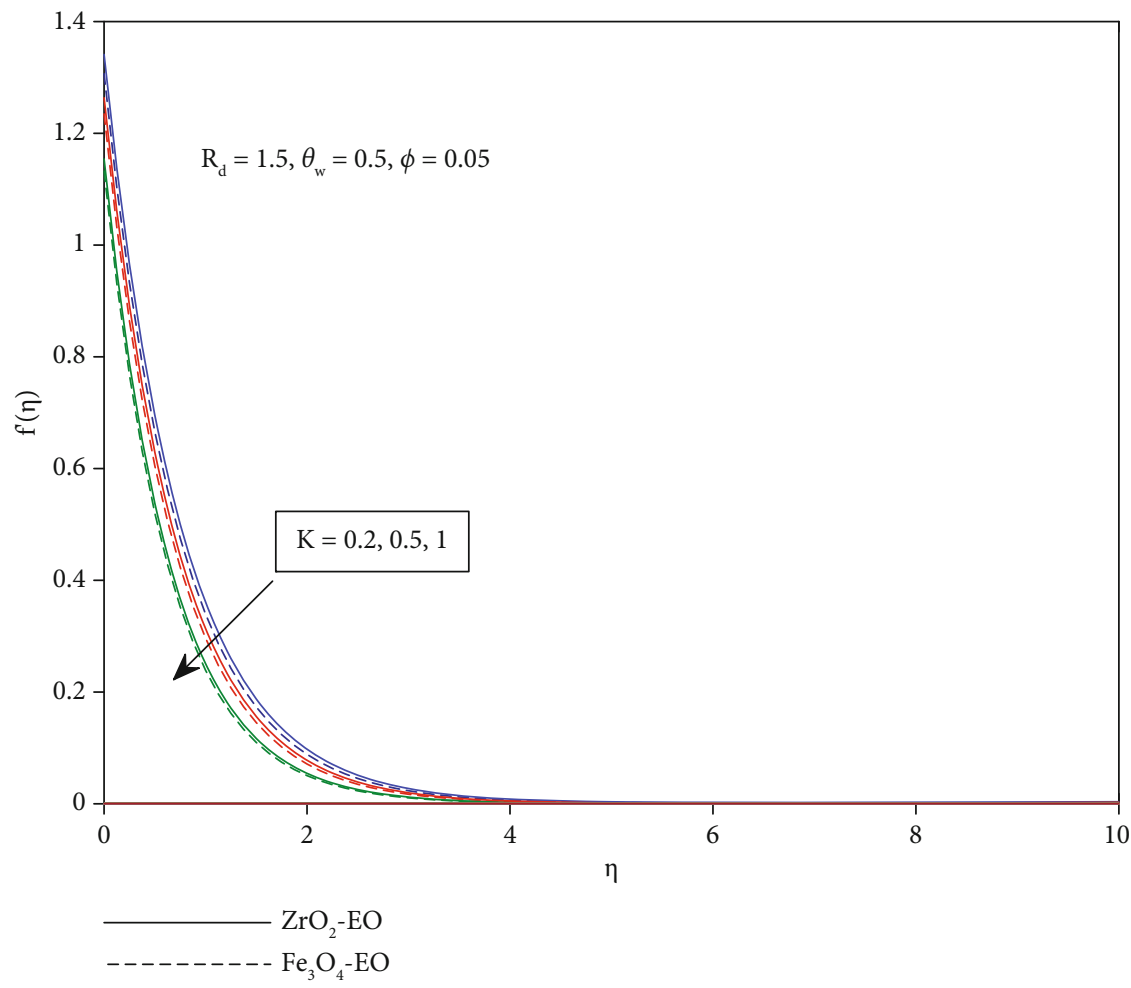

FIGURE 1: Velocity profile for $K$.

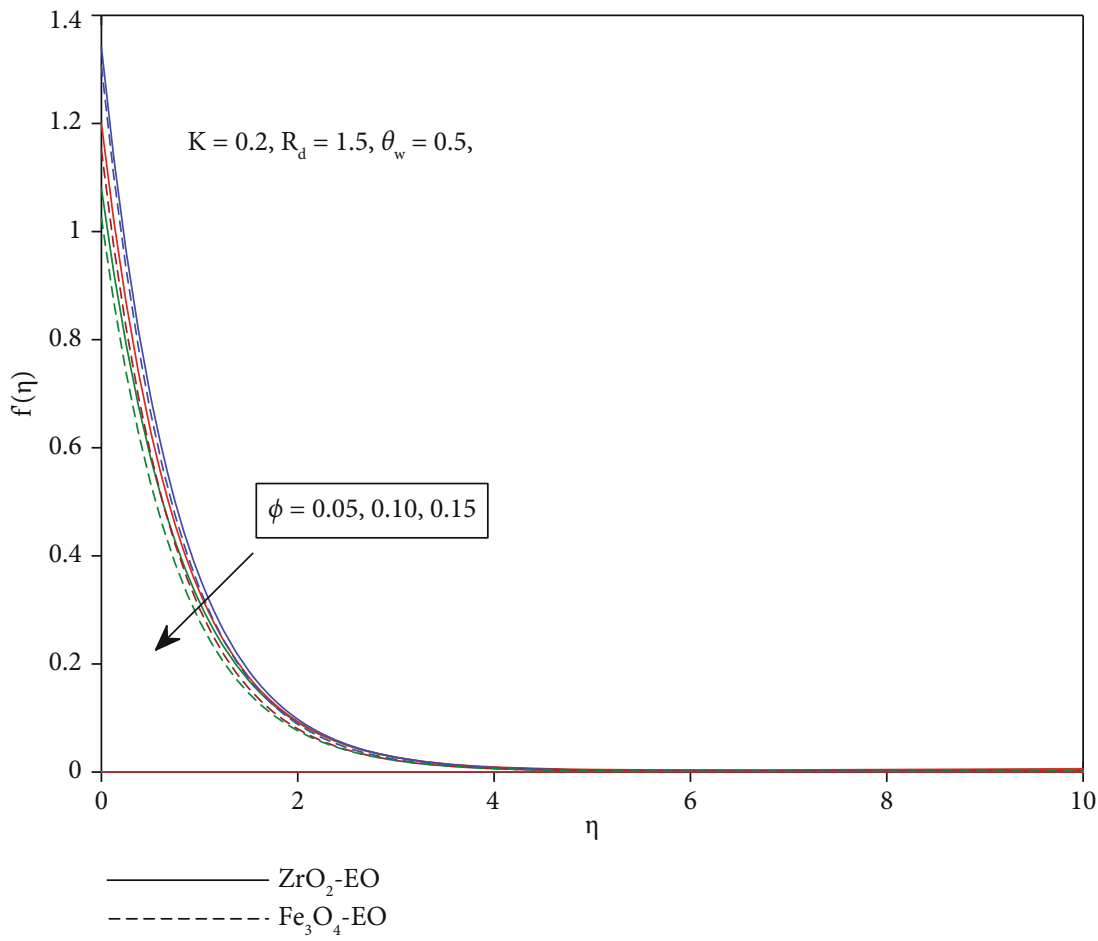

FIgURE 2: Velocity profile for $\phi$. 


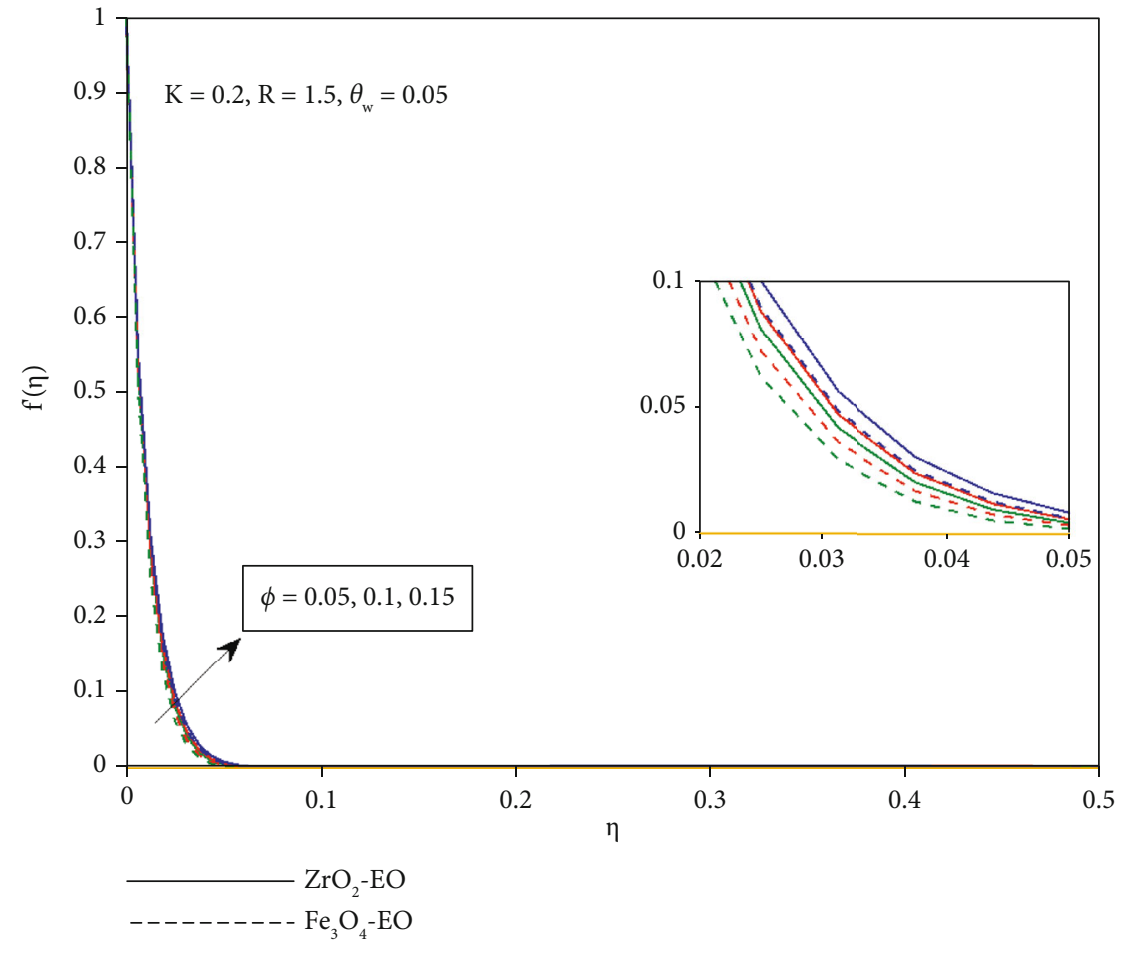

Figure 3: Temperature profile for $\phi$.

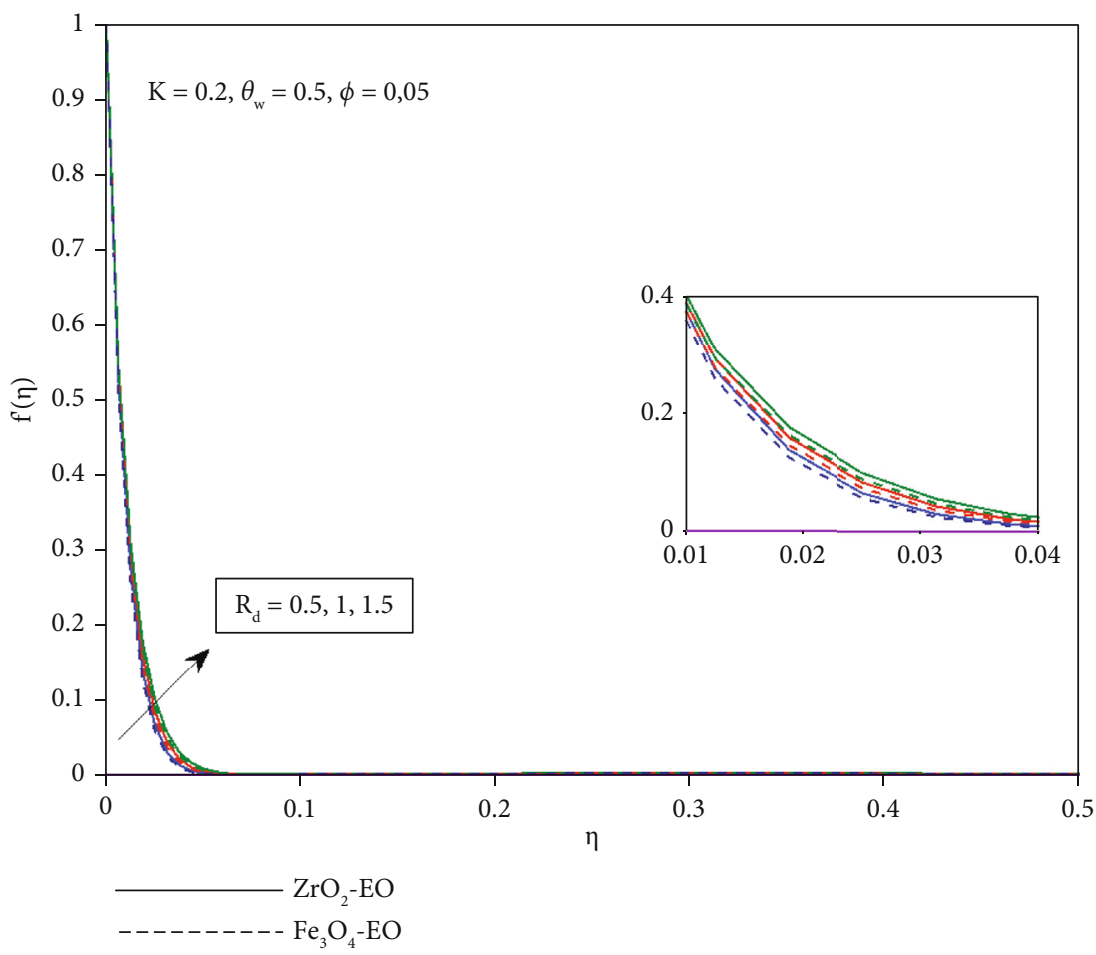

Figure 4: Temperature profile for $R_{d}$. 


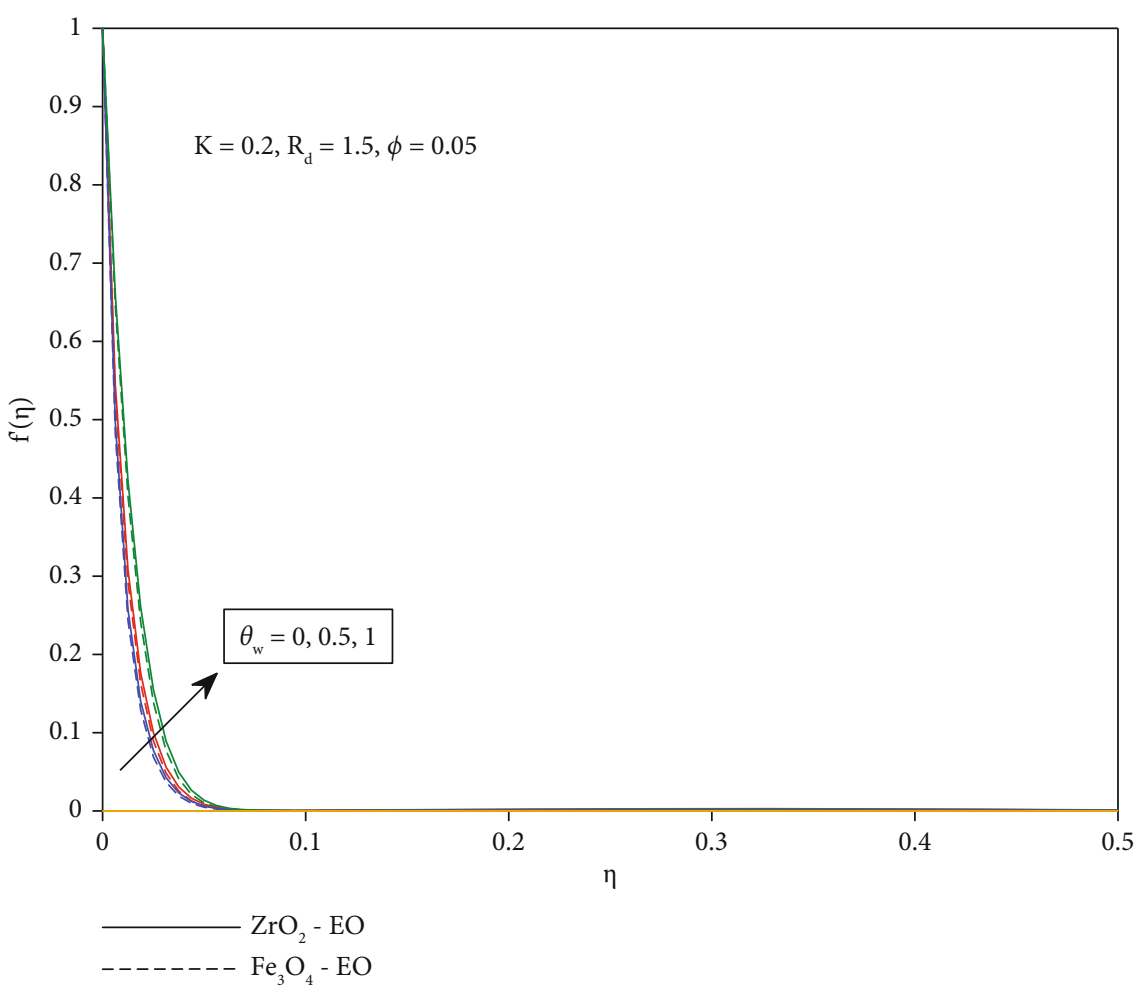

Figure 5: Temperature profile for $\theta_{w}$.

TABle 3: Comparison for different values of the Prandtl number for $-\theta^{\prime}(0)$ assuming $R_{d}=K=\phi=\theta_{w}=0$.

\begin{tabular}{lccc}
\hline $\operatorname{Pr}$ & Jamshed et al. [4] & Das et al. [21] & Present study \\
\hline 0.72 & 0.80876181 & 0.80876122 & 0.80876183 \\
1 & 1 & 1 & 1 \\
3 & 1.92357420 & 1.92357431 & 1.92357424 \\
7 & 3.07314651 & 3.07314679 & 3.07314652 \\
10 & 3.72055429 & 3.72055436 & 3.72055430 \\
\hline
\end{tabular}

\section{Conclusions}

Marangoni convection of $\mathrm{Fe}_{3} \mathrm{O}_{4}$ and $\mathrm{ZrO}_{2}$ nanofluid flow past a stretching surface with thermal radiation embedded in porous media is considered. The flow's governing model is encountered with the Runge-Kutta fourth-order technique and suitable similarity transformations. These results can be used to study the thermal performance with other boundary conditions.

The following important outcomes are obtained.

(i) The $\mathrm{Fe}_{3} \mathrm{O}_{4} / \mathrm{EO}$ nanofluid is more significant in the cooling process in comparison to the $\mathrm{ZrO}_{2} / \mathrm{EO}$ nanofluid

(ii) With increased porosity parameter $K$, a decrement in the velocity field has been noticed for both nanofluids (iii) With increased $R_{d}$ and $\theta_{w}$ parameters, a decrement in the temperature field has been noticed for both nanofluids

(iv) For the increased values of $\phi$, a decrement is noticed for the velocity profile and opposite behavior is observed for temperature profiles for $\mathrm{Fe}_{3} \mathrm{O}_{4} / \mathrm{EO}$ and $\mathrm{ZrO}_{2} / \mathrm{EO}$ nanofluids

\section{Data Availability}

No data were used to support this study.

\section{Conflicts of Interest}

There is no conflict of interest regarding the publication of this article.

\section{References}

[1] S. U. S. Choi, "Enhancing thermal conductivity of fluids with nanoparticles," in The Proceedings of the 1995 ASME International Mechanical Engineering Congress and Exposition, pp. 99-105, San Francisco, ASME, FED 231/MD66, USA, 1995.

[2] Y. M. Xuan and W. Roetzel, "Conceptions of heat transfer correlation of nanofluids," International Journal Communications of Heat and Mass Transfer, vol. 43, pp. 3701-3707, 2020.

[3] L. A. Khan, M. Raza, N. A. Mir, and R. Ellahi, "Effects of different shapes of nanoparticles on peristaltic flow of MHD 
nanofluids filled in an asymmetric channel," Journal of Thermal Analysis and Calorimetry, vol. 140, pp. 879-890, 2020.

[4] W. Jamshed, K. S. Nisar, R. W. Ibrahim, T. Mukhtar, V. Vijayakumar, and F. Ahmad, "Computational frame work of Cattaneo-Christov heat flux effects on engine oil based Williamson hybrid nanofluids: a thermal case study," Case Studies in Thermal Engineering, vol. 26, 2021.

[5] P. K. Dadheech, P. Agrawal, F. Mebarek-Oudina, N. Abu-Hamdeh, and A. Sharma, "Comparative heat transfer analysis of $\mathrm{MoS} 2 / \mathrm{C} 2 \mathrm{H} 6 \mathrm{O} 2$ and $\mathrm{SiO} 2-\mathrm{MoS} 2 /$ $\mathrm{C} 2 \mathrm{H} 6 \mathrm{O} 2 \mathrm{Nanofluids}$ with natural convection and inclined magnetic field," Journal of Nanofluids, vol. 9, no. 3, pp. 161-167, 2020.

[6] A. Mourad, A. Aissa, F. Mebarek-Oudina et al., "Galerkin finite element analysis of thermal aspects of $\mathrm{Fe}_{3} \mathrm{O}_{4}$ MWCNT/water hybrid nanofluid filled in wavy enclosure with uniform magnetic field effect," International Communications in Heat and Mass Transfer, vol. 126, 2021.

[7] A. Saeed, P. Kumam, S. Nasir, T. Gul, and W. Kumam, "Nonlinear convective flow of the thin film nanofluid over an inclined stretching surface," Scientific Reports, vol. 11, no. 1, 2021.

[8] M. Bilal, A. Saeed, T. Gul, I. Ali, W. Kumam, and P. Kumam, "Numerical approximation of microorganisms hybrid nanofluid flow induced by a wavy fluctuating spinning disc," Coatings, vol. 11, no. 9, p. 1032, 2021.

[9] A. Saeed, P. Kumam, T. Gul, W. Alghamdi, W. Kumam, and A. Khan, "Darcy-Forchheimer couple stress hybrid nanofluids flow with variable fluid properties," Scientific Reports, vol. 11, no. 1, 2021.

[10] R. Ellahi, F. Hussain, S. Asad Abbas, M. M. Sarafraz, M. Goodarzi, and M. S. Shadloo, "Study of two-phase Newtonian nanofluid flow hybrid with hafnium particles under the effects of slip," Inventions, vol. 5, no. 1, p. 6, 2020.

[11] M. Raza, R. Ellahi, S. M. Sait et al., "Enhancement of heat transfer in peristaltic flow in a permeable channel under induced magnetic field using different CNTs," Journal of Thermal Analysis and Calorimetry, vol. 140, pp. 1277-1291, 2020.

[12] P. K. Dadheech, P. Agrawal, A. Sharma, A. Dadheech, Q. alMdallal, and S. D. Purohit, "Entropy analysis for radiative inclined MHD slip flow with heat source in porous medium for two different fluids," Case Studies in Thermal Engineering, vol. 28, 2021.

[13] K. S. Nisar, U. Khan, A. Zaib, I. Khan, and D. Baleanu, "Numerical Simulation of Mixed Convection Squeezing Flow of a Hybrid Nanofluid Containing Magnetized Ferroparticles in 50\%:50\% of Ethylene Glycol-Water Mixture Base Fluids Between Two Disks With the Presence of a Non-linear Thermal Radiation Heat Flux," Frontiers in Chemistry, vol. 8, p. 792, 2020.

[14] L. G. Napolitano, "Marangoni boundary layers," in Proceedings 3rd European Symposium on Material Science in Space, Grenoble, 1979.

[15] D. M. Christopher and B. X. Wang, "Prandtl number effects for Marangoni convection over a flat surface," International Journal of Thermal Sciences, vol. 40, no. 6, pp. 564-570, 2001.

[16] E. H. Aly and A. Ebaid, "Exact analysis for the effect of heat transfer on MHD and radiation Marangoni boundary layer nanofluid flow pasta surface embedded in a porous medium," Journal of Molecular Liquids, vol. 215, 2016.
[17] B. Yang, M. M. Sarafraz, and M. Arjomandi, "Marangoni effect on the thermal performance of glycerol/water mixture in microchannel," Applied Thermal Engineering, vol. 161, 2019.

[18] Q. M. Al-Mdallal, N. Indumathi, B. Ganga, and A. K. Abdul Hakeem, "Marangoni radiative effects of hybrid-nanofluids flow past a permeable surface with inclined magnetic field," Case Studies in Thermal Engineering, vol. 17, 2020.

[19] P. K. Dadheech, P. Agrawal, A. Sharma, K. S. Nisar, and S. D. Purohit, "Marangoni convection flow of $\gamma-\mathrm{Al}_{2} \mathrm{O}_{3}$ nanofluids past a porous stretching surface with thermal radiation effect in the presence of an inclined magnetic field," Heat Transfer, vol. 51, no. 1, pp. 534-550, 2022.

[20] P. Agrawal, P. K. Dadheech, R. N. Jat, K. S. Nisar, M. Bohra, and S. D. Purohit, "Magneto Marangoni flow of $\gamma$-AL2O3 nanofluids with thermal radiation and heat source/sink effects over a stretching surface embedded in porous medium," Case Studies in Thermal Engineering, vol. 23, 2021.

[21] S. Das, S. Chakraborty, R. N. Jana, and O. D. Makinde, "Entropy analysis of unsteady magneto-nanofluid flow past accelerating stretching sheet with convective boundary condition," Applied Mathematics and Mechanics, vol. 36, no. 12, pp. 1593-1610, 2015. 